\title{
Object-Based Image Analysis based on A Region-Line Primitive Association Framework
}

\author{
M. Wang and J. Wang \\ Key Laboratory of Virtual Geographic Environment (Nanjing Normal University), Ministry of Education, Nanjing, Jiangsu, P.R. \\ China, 210023 -sysj0918@126.com; wangjieyl@sina.com
}

KEY WORDS: Region, Straight Line, Primitive, Image Segmentation, Image Classification, Road Extraction

\section{INTRODUCTION}

Common object-based image analysis (OBIA) applications generally follow the "segment and then classify" framework. However, information extraction based only on regions has many limitations. For example, image edges are not considered during common OBIAs. Image edge lines generally denote object boundaries or borders, which exhibit strong semantic connotations. However, region boundaries obtained by image segmentation do not necessarily match image edge lines in quantity and location. Thus, image edges should be additionally used in OBIA. In this study, straight lines are used in our OBIA framework for extracting man-made objects, because the latter generally have straight line-shaped boundaries.

The current study introduces a region-line primitive association framework (RLPAF) for OBIA. Regions and lines are used collaboratively from low-level image processing (segmentation) and feature extraction to high-level image analysis (classification and recognition). Several region-line associating techniques for analysing object shapes and relationships were designed. Our techniques have been validated by impervious surface and road network extraction from high spatial resolution (HSR) images.

\section{METHODS}

\subsection{Technical Framework of RLPAF}

In Fig. 1 which illustrates RLPAF, HBC-SEG and Burn's phase-grouping method are used to obtain the region primitives and straight line primitives. Region and line features, including the spectra and shapes of the regions as well the lengths and directions of the lines are calculated. The association model RLPAF is built based on region and line topologies and orientation relationships. Thematic information extraction, image classification, and change detection can then be conducted by both considering the two kinds of primitives.

In RLPAF, direction relationships of a region to a line are classified as "above" "bilateral" and "below". The topology of a region to a line includes separation, intersection, tangent, and inclusion. The combination of different direction and topology relationships creates several region-line relationships, which can be used as new features for OBIAs.

In this study, a set of line-based concepts is proposed and used in subsequent OBIAs, which include:

-Unilateral and Tangent Relationship

- Ipsilateral Neighbors
- Ipsilateral and Homogeneous Chain

- Line-Based Length-to-Width Ratio

- Region and Line Mutual Conversion Operators

Figure 3 illustrates the concept of line-based length-to-Width Ratio (LBLW). The width of the region is defined by its tangent straight line by moving the line along its perpendicular direction and record the span where the touching length declines dramatically. A region can have multiple LBLW measures based on its multiple tangent straight lines, which thus describes irregular shapes objectively and precisely.

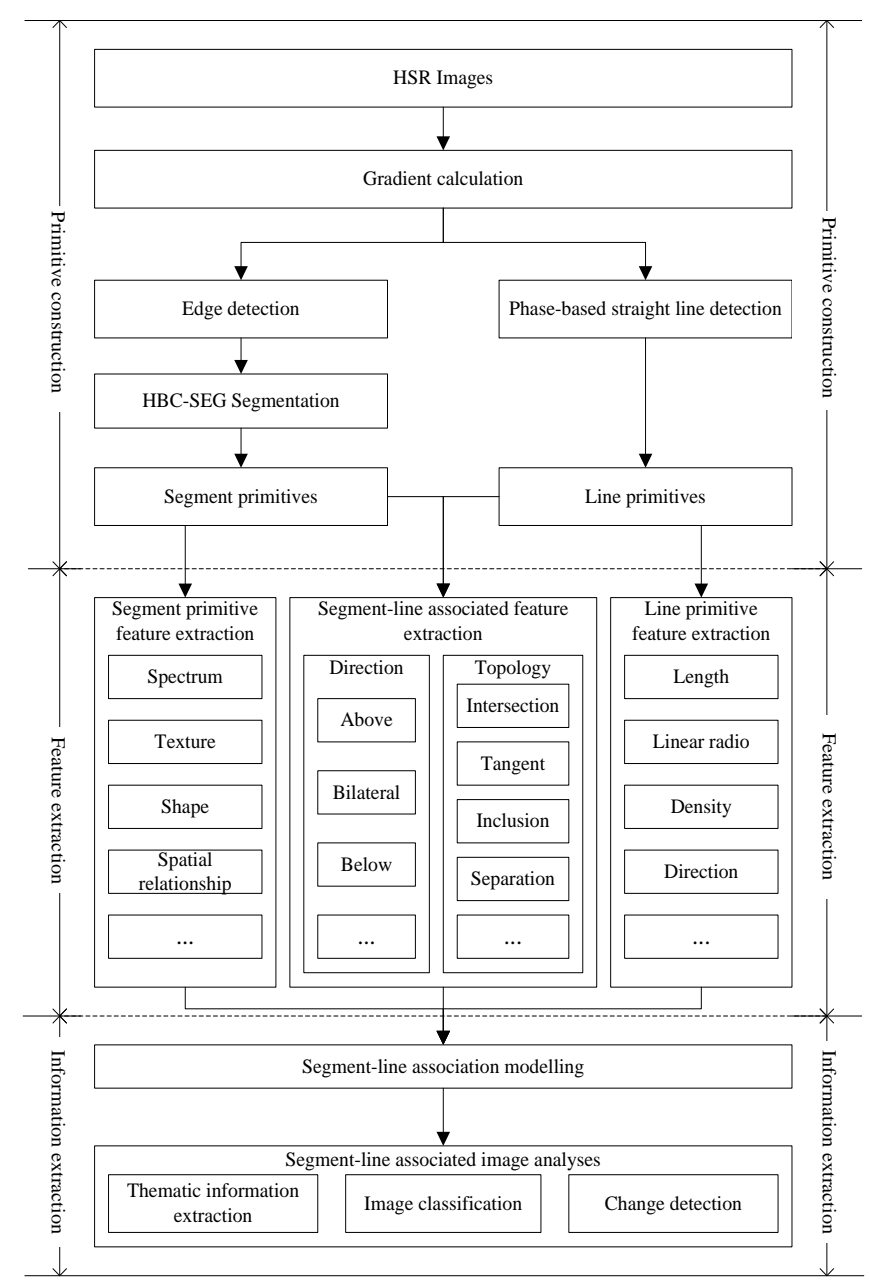

Figure 1. Technical framework. 


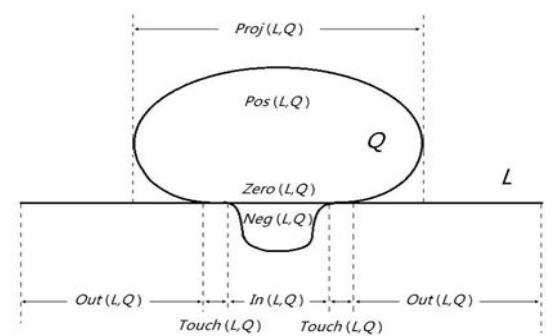

(a)
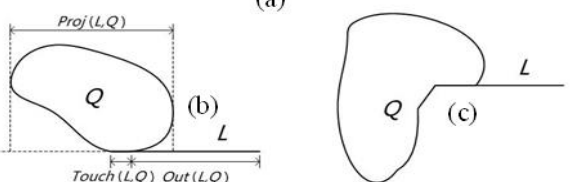

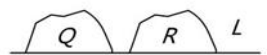

(d)
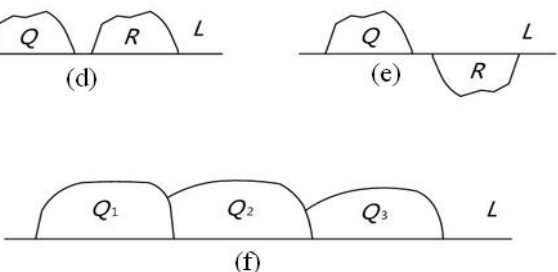

Figure 2. Region-line relationship modeling. (a) A pair of touching region and line. (b) A unilateral case. (c) A tangent relationship that is not unilateral. (d) An IPSL-neighborhood relationship. (e) Regions that are not IPSL-neighbors because they are located bilaterally to line $L$. (f) An IPSL chain.

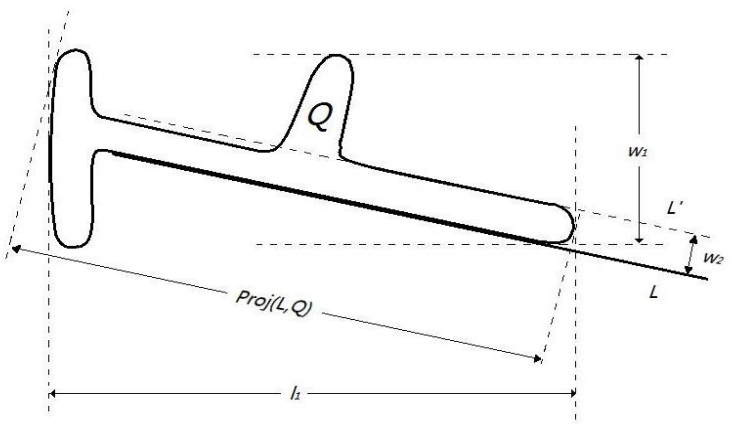

Figure 3. Line-based length-to-width ratio.

\subsection{RLPAF-based road-extraction}

An RLPAF-based road-extraction method was designed to exemplify and validate PRLAF (Figure 4). First-level, coarsegrained supervised classification based on region spectral signatures was initially performed to separate impervious surfaces. After that, second-level rule-based classification extracts high $L B L W$ segments within an impervious surface as roads. After that, a straight line-guided depth-searching step was designed to extend initial, broken road regions into a road network. This searching step involves several region-to-line, line-to-line, and line-to-region conversions. Road regions with low $L B L W \mathrm{~s}$ and sufficient line-based widths $(L B W \mathrm{~s})$ are thus appended into the initial road segments and formed the final road network.

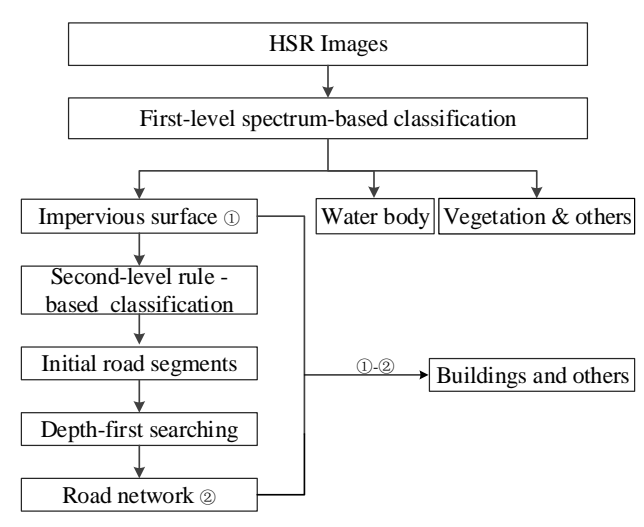

Figure 4. Impervious surface and road extraction scheme.

\section{EXPERIMENTS}

The methods were applied in different HSR images for validation. In the experiments, impervious surfaces were first extracted from HSR images by first-level supervised classification using region spectral features. Then, three road extraction schema, i.e., using common $L W$ measure (the $L W$ method), $L B L W$ measure (the LBLW method), and $L B L W$ measure combined with depth searching (the LBLW\&DS method), were applied on the impervious surface to extract road regions with high $L W \mathrm{~s}$ or $L B L W \mathrm{~s}$. Accuracy measures, including Recall, Precision, and F-measure, were employed to evaluate road-extraction accuracy.

In the experiments, given a length-to-width threshold $T$, the recall ratios of the three methods were LBLW\&DS > LBLW > LW, whereas their precision measures were reversed as LW $>$ LBLW > LBLW\&DS. However, the LBLW and LBLW\&DS methods obviously performed better than the LW method on the $F$-measure, given the same threshold $T$. The compensation on recall ratio far exceeded that on precision loss when the LBLW and LBLW\&DS methods were compared with the LW method. In addition, the LBLW\&DS method had flat $F$-measure curves in all the experiments.

In Figure 5, by visual interpretation, the LW method retrieved the minimum road regions and formed a broken road network. The LBLW and LBLW\&DS methods obtained significantly denser road regions and formed continuous road networks while inducing additional errors. As illustrated in Fig. 5(g), in a zoomed-in section of the experimental area, broken roads are connected by the depth-searching process of the LBLW\&DS method.

In all the experiments, straight lines played at least two important roles. 1) Region shape analysis became more accurate than that in common region only-based OBIA. 2) The linebased depth-searching process offered precise directions and the capability to override gaps. The LBLW and LBLW\&DS methods obviously performed better than the LW method on both the quantitative and qualitative analyses.

In conclusion, the proposed framework and methods refine primitive shape and spatial relationship analyses, as well as obtain higher method accuracy, than OBIAs based on only regions. However, the extracted road network exhibited many defects and might only serve as an initial extracted result for subsequent refinement. Possible segmentation and classification errors, as well as the simplicity of current road-extraction rules 
are the main causes of these defects. Sophisticated OBIA rules or processing are necessary to improve the accuracy of road extraction particularly in urban areas. Nevertheless, the proposed scheme can serve as an initial step for road extraction because of its simplicity and effectiveness.
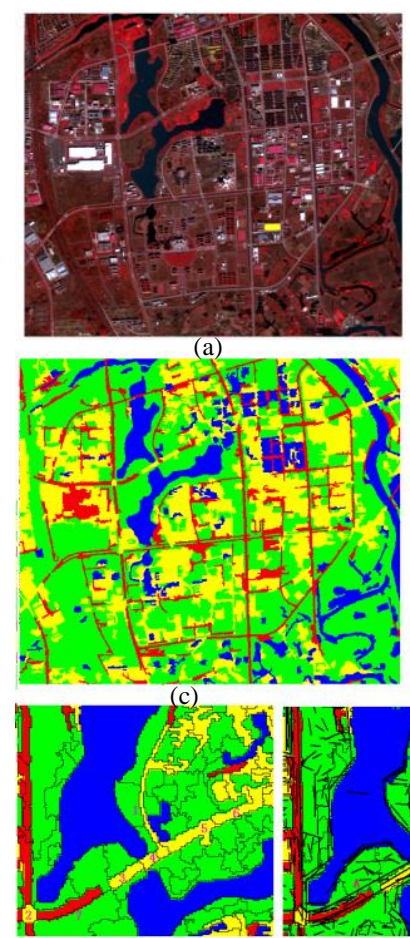

(e)
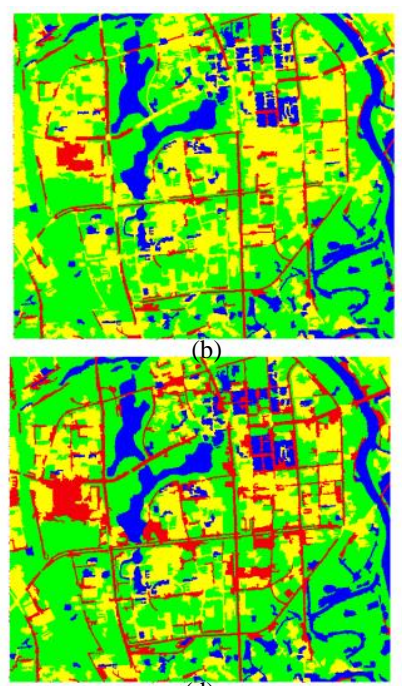

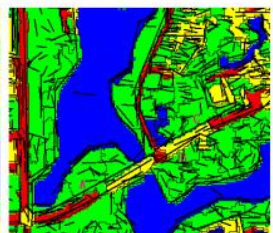

(f)

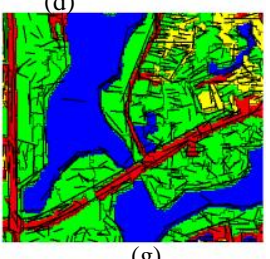

(g)

Figure 5. Experimental area. (a) Original data. (b), (c), and (d) The red regions are roads extracted by the LW, LBLW, and LBLW\&DS methods, respectively. (e), (f), and (g) The zoomedin images of (b), (c), and (d), respectively.

\section{CONCLUSTIONS}

RLPAF comprehensively utilizes line and region primitives in OBIA by image segmentation, straight line detection, and region-line relationship modelling. The proposed framework is then applied and validated in extracting road networks from HSR images. In the road extraction task, region shape analysis and spatial relationship reasoning are facilitated by considering both region and line primitives. In future work, we will investigate to extend the proposed framework and methods, as well as optimizing their performance in extensive application scenarios.

\section{ACKNOWLEDGMENT}

This work is jointly supported by the Natural Science Foundation of Jiangsu Province, China (BK20140042), the Qing Lan Project, and the Priority Academic Program Development of Jiangsu Higher Education Institutions.

P.S. A paper version of this abstraction can be found in "M. Wang and J. Wang. A Region-Line Primitive Association Framework for Object-Based Remote Sensing Image Analysis. Photogrammetric engineering \& Remote sensing, 2016, 82(2):149-159." Some of these figures used in the current abstract are from or modified from the aforementioned paper. 